Proyecciones Journal of Mathematics

Vol. 37, No 3, pp. 503-517, September 2018.

Universidad Católica del Norte

Antofagasta - Chile

\title{
The $t$-pebbling number of Lamp graphs
}

\author{
A. Lourdusamy \\ St. Xavier's College (Autonomous), India \\ F. Patrick \\ St. Xavier's College (Autonomous), India \\ and \\ T. Mathivanan \\ St. Xavier's College (Autonomous), India \\ Received: September 2017. Accepted : January 2018
}

\begin{abstract}
Let $G$ be a graph and some pebbles are distributed on its vertices. A pebbling move (step) consists of removing two pebbles from one vertex, throwing one pebble away, and moving the other pebble to an adjacent vertex. The t-pebbling number of a graph $G$ is the least integer $m$ such that from any distribution of $m$ pebbles on the vertices of $G$, we can move t pebbles to any specified vertex by a sequence of pebbling moves. In this paper, we determine the t-pebbling number of Lamp graphs.
\end{abstract}

Keywords : Pebbling number, t-Pebbling number, Lamp graphs.

AMS Subject Classification 2010 : 05C99. 


\section{Introduction}

Pebbling in graphs was first considered by Chung [1]. Graph Pebbling is a network optimization model for the transportation of resources that are consumed in transit. The central problem in this model asks whether discrete pebbles from one set of vertices can be moved to another while pebbles are lost in the process. The graph pebbling model was born as a method for solving a combinatorial number theory conjecture of Paul Erdős $[2,3]$. Lemke [4] has given different version of the conjecture. The conjecture has got applications to problems in combinatorial group theory and $p$-adic diophantine equations. Here, the term graph refers to a simple graph. A configuration $C$ of pebbles on a graph $G=(V, E)$ can be thought of as a function $C: V(G) \rightarrow N \cup\{0\}$. The value $C(v)$ equals the number of pebbles placed at vertex $v$, and the size of the configuration is the number $|C|=\sum_{v \in V(G)} C(v)$ of pebbles placed in total on $G$. Suppose $C$ is a configuration of pebbles on a graph G. A pebbling move (step) consists of removing two pebbles from one vertex and then placing one pebble at an adjacent vertex. We say a pebble can be moved to a vertex $v$, the target vertex, if we can apply pebbling moves repeatedly (if necessary) so that in the resulting configuration the vertex $v$ has at least one pebble.

Definition 1.1. [5] The pebbling number of a vertex $v$ in a graph $G, f(v, G)$, is the smallest positive integer $m$ such that however $m$ pebbles are placed on the vertices of the graph, a pebble can be moved to $v$ in finite number of pebbling moves, each move removes two pebbles of one vertex and placing one on an adjacent vertex. The pebbling number of $G, f(G)$, is defined to be the maximum of the pebbling numbers of its vertices.

Thus the pebbling number of a graph $G, f(G)$, is the least $m$ such that, for any configuration of $m$ pebbles to the vertices of $G$, we can move a pebble to any vertex by a sequence of moves, each move removes two pebbles of one vertex and placing one on an adjacent vertex.

Definition 1.2. [5] The t-pebbling number of a vertex $v$ in a graph $G$, $f_{t}(v, G)$, is the smallest positive integer $m$ such that however $m$ pebbles are placed on the vertices of the graph, $t$ pebbles can be moved to $v$ in finite number of pebbling moves, each move removes two pebbles of one vertex and placing one on an adjacent vertex. The t-pebbling number of 
$G, f_{t}(G)$, is defined to be the maximum of the pebbling numbers of its vertices.

Thus the t-pebbling number of a graph $G, f_{t}(G)$, is the least $m$ such that, for any configuration of $m$ pebbles to the vertices of $G$, we can move $t$ pebbles to any vertex by a sequence of moves, each move removes two pebbles of one vertex and placing one on an adjacent vertex.

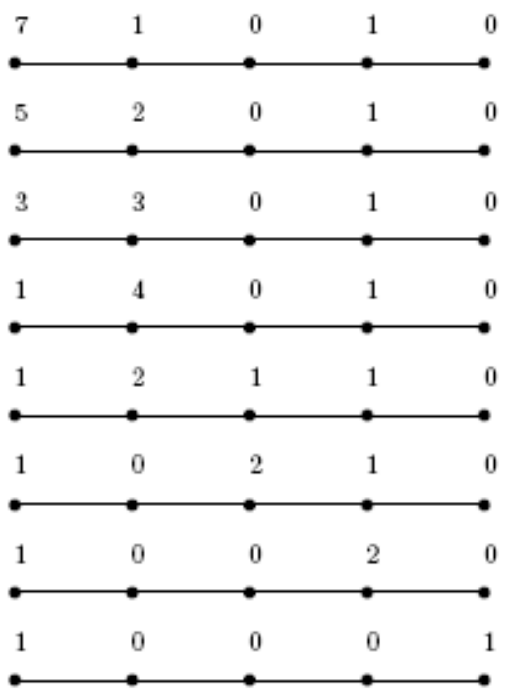

Figure 1. An illustration of moving one pebble to the end vertex of the path $P_{5}$ from a configuration of size 9 .

Fact 1.3. [12] For any vertex $v$ of a graph $G, f(v, G) \geq n$ where $n=$ $|V(G)|$.

Fact 1.4. [12] The pebbling number of a graph $G$ satisfies

$$
f(G) \geq \max \left\{2^{\operatorname{diam}(G)},|V(G)|\right\} .
$$

Now, we state the known pebbling results of the Jahangir graph $J_{2, m}$ which will be used to prove the results of Section 2 .

Definition 1.5. [11] Jahangir graph $J_{n, m}$ for $m \geq 3$ is a graph on $n m+1$ vertices, consisting of a cycle $C_{n m}$ with one additional vertex which is adjacent to $m$ vertices of $C_{n m}$ at distance $n$ to each other on $C_{n m}$. 
The pebbling number of Jahangir graph $J_{2, m}(m \geq 3)$ is as follows:

Theorem 1.6. For the Jahangir graph $J_{2,3}, f\left(J_{2,3}\right)=8$.

Theorem 1.7. For the Jahangir graph $J_{2,7}, f\left(J_{2,7}\right)=23$.

Theorem 1.8. For the Jahangir graph $J_{2, m}$ where $m \geq 8, f\left(J_{2, m}\right)=2 m+$ 10 .

Lourdusamy et al. determined the $t$-pebbling number of Jahangir graph $J_{3, m}$ (for $m \geq 3$ ) in [5]. And also they determined the $t$-pebbling number for squares of cycles $(t \geq 2)$ in [6] and for some wheel related graphs in [8].

Remark 1.9. Consider a graph $G$ with $n$ vertices and $f(G)$ pebbles are placed on its vertices. Suppose we choose a target vertex $v$ from $G$ to put a pebble on it. If $C(v) \geq 1$ or $C(u) \geq 2$ where $u v \in E(G)$, then we can move one pebble to $v$ easily. So, we always assume that $C(v)=0$ and $C(u) \leq 1$ for all $u v \in E(G)$ when $v$ is the target vertex.

Lemma 1. If $H$ is a spanning connected subgraph of $G$, then $f_{t}(G) \leq$ $f_{t}(H)$.

Proof. Let $x$ be the $t$-pebbling number of the graph $H$. Obviously, $G$ may or may not contain some more edges than $H$. Clearly, adding the remaining edges of $G$ to $H$ will not increase the $t$-pebbling number of $G$. Thus $f_{t}(G) \leq x=f_{t}(H)$.

In the next section, we define the Lamp graph and then determine its $t$-pebbling number. 


\section{The $t$-pebbling number of Lamp graphs}

Definition 2.1. The join $G+H$ of two graphs $G$ and $H$ is the graph with vertex set $V(G+H)=V(G) \cup V(H)$ and edge set $E(G+H)=$ $E(G) \cup E(H) \cup\{u v: u \in V(G), v \in V(H)\}$.

Definition 2.2. The wheel $W_{n}$ is defined as the join $C_{n}+K_{1}$. The vertex $K_{1}$ is the apex vertex and the vertices on the underlying cycle are called rim vertices. The edges of the underlying cycle are called the rim edges and the edges joining the apex and the rim vertices are called spoke edges.

Definition 2.3. The Lamp graph is obtained from the wheel graph $W_{n}$ $(n \geq 3)$ by adding a new vertex corresponding to each rim edge of the wheel and make the new vertex adjacent to end vertices of corresponding rim edge. We denote the Lamp graph by $L_{n}$.

Labeling for $L_{n}$ : Let $n \geq 3$. Let $v_{2 n+1}$ be the label of the center vertex and then label the other vertices of $L_{n}$ by $v_{1}, v_{2}, \cdots, v_{2 n}$ such that $\operatorname{deg}\left(v_{1}\right)=5$, $\operatorname{deg}\left(v_{2}\right)=2, \cdots \operatorname{deg}\left(v_{2 n-1}\right)=5, \operatorname{deg}\left(v_{2 n}\right)=2$. An illustration of the Lamp graph $L_{8}$ is shown in Figure 2.

We define the sets $S_{1}=\left\{v_{1}, v_{3}, \cdots, v_{2 n-1}\right\}$ and $S_{2}=\left\{v_{2}, v_{4}, \cdots, v_{2 n}\right\}$ from the labeling of $L_{n}$.

Theorem 2.4. For $L_{3}$, the $t$-pebbling number is $f_{t}\left(L_{3}\right)=4(t-1)+8$.

Proof. Let $C\left(v_{4}\right)=4(t-1)+3, C\left(v_{6}\right)=3, C\left(v_{7}\right)=1$ and $C\left(v_{i}\right)=0$ for all $i \neq 4,6,7$. Then we cannot move $t$ pebbles to $v_{2}$. Thus $f_{t}\left(L_{3}\right) \geq$ $4(t-1)+8$.

Since $J_{2,3}$ is a spanning subgraph of $L_{3}$ and by Lemma 1, we have $f\left(L_{3}\right) \leq f\left(J_{2,3}\right)=8$ (Theorem ??). So the result is true for $t=1$. Assume the result is true for $t^{\prime} \geq 2$. Now consider the distribution of $4(t-1)+8$ pebbles on the vertices of $L_{3}$. Clearly, we can move one pebble to any target vertex $v_{i}$ at a cost of at most four pebbles, since $C\left(L_{3}\right) \geq 12$ and $f\left(L_{3}\right)=8$. After moving one pebble to $v_{i}$, the remaining number of pebbles on the vertices of $L_{3}$ is at least $4(t-2)+8$. Hence we can move the additional $t-1$ pebbles to $v_{i}$, by induction. Thus $f_{t}\left(L_{3}\right) \leq 4(t-1)+8$. 
Theorem 2.5. For $L_{4}$, the $t$-pebbling number is $f_{t}\left(L_{4}\right)=8(t-1)+11$.

Proof. Let $C\left(v_{6}\right)=8(t-1)+7, C\left(v_{4}\right)=C\left(v_{8}\right)=C\left(v_{9}\right)=1$ and $C\left(v_{i}\right)=0$ for all $i \neq 4,6,8,9$. Then we cannot move $t$ pebbles to $v_{2}$. Thus $f_{t}\left(L_{4}\right) \geq 8(t-1)+11$.

We have three cases to prove $f\left(L_{4}\right) \leq 11$. Consider the distribution of 11 pebbles on the vertices of $L_{4}$.

Case 1: Let $v_{9}$ be the target vertex.

Clearly, $C\left(v_{9}\right)=0$ and $C\left(v_{i}\right) \leq 1$ for all $v_{i} \in S_{1}$ by Remark 1.9. Since, $C\left(S_{2}\right) \geq 7$, there exists a vertex, say $v_{2}$, such that $C\left(v_{2}\right) \geq 2$. If $C\left(v_{1}\right)=1$ or $C\left(v_{3}\right)=1$ or $C\left(v_{4}\right) \geq 2$ or $C\left(v_{8}\right) \geq 2$ then we can move one pebble to $v_{9}$ easily. Assume $C\left(v_{1}\right)=0, C\left(v_{3}\right)=0, C\left(v_{4}\right) \leq 1$, and $C\left(v_{8}\right) \leq 1$. Now, we can move one pebble to $v_{9}$ easily, since $C\left(v_{2}\right) \geq 4$ or $C\left(v_{6}\right) \geq 4$.

Case 2: Let $v_{1}$ be the target vertex.

Clearly, $C\left(v_{1}\right)=0, C\left(v_{2}\right) \leq 1, C\left(v_{3}\right) \leq 1, C\left(v_{7}\right) \leq 1, C\left(v_{8}\right) \leq 1$ and $C\left(v_{9}\right) \leq 1$, by Remark 1.9. Let $C\left(v_{5}\right) \geq 2$. If $C\left(v_{5}\right) \geq 4$, then clearly we are done. If $C\left(v_{5}\right)=2$ or 3 then either $C\left(v_{4}\right) \geq 2$ or $C\left(v_{6}\right) \geq 2$. So, we can move two pebbles to $v_{3}$ or $v_{7}$ and hence one pebble can be moved to $v_{1}$. Assume $C\left(v_{5}\right) \leq 1$. Without loss of generality, we assume $C\left(v_{4}\right) \geq 3$. If $C\left(v_{4}\right) \geq 4$ then clearly we are done. Assume $C\left(v_{4}\right)=3$ and hence $C\left(v_{6}\right) \geq 2$. Clearly we are done if $C\left(v_{5}\right)=1$ or $C\left(v_{7}\right)=1$. Assume $C\left(v_{5}\right)=C\left(v_{7}\right)=0$ and hence we get $C\left(v_{6}\right) \geq 4$. Thus we can move one pebble to $v_{1}$ easily.

Case 3: Let $v_{2}$ be the target vertex.

Clearly, $C\left(v_{2}\right)=0, C\left(v_{1}\right) \leq 1$ and $C\left(v_{3}\right) \leq 1$, by Remark 1.9. Let $C\left(v_{4}\right) \geq$ 2. If $C\left(v_{4}\right) \geq 4$ then clearly we are done. Assume $C\left(v_{4}\right)=2$ or 3 then clearly $C\left(v_{3}\right)=0, C\left(v_{5}\right) \leq 1$ and $C\left(v_{9}\right) \leq 1$ (otherwise, we can move one pebble to $v_{2}$ ). If $C\left(v_{7}\right) \geq 4$ or $C\left(v_{8}\right) \geq 4$ or $C\left(v_{7}\right) \geq 2$ and $C\left(v_{8}\right) \geq 2$ then we can move two pebbles to $v_{1}$ and then we move one pebble to $v_{2}$ from $v_{1}$. So, we assume $C\left(v_{1}\right)+C\left(v_{7}\right)+C\left(v_{8}\right) \leq 4$ such that we cannot move one pebble to $v_{2}$. This implies that $C\left(v_{6}\right) \geq 2$. Clearly we are done if $C\left(v_{5}\right)=1$. Let $C\left(v_{7}\right) \geq 2$. Thus we can move one pebble to $v_{1}$ from $v_{7}$. If $C\left(v_{9}\right)=1$ then we can move another one pebble to $v_{1}$ using the pebbles 
at $v_{4}$ and $v_{6}$. If $C\left(v_{9}\right)=0$ then we get $C\left(v_{6}\right) \geq 4$ and hence we can move another one pebble to $v_{1}$ using the pebbles at $v_{6}$. Assume $C\left(v_{7}\right) \leq 1$. In a similar way, we assume $C\left(v_{8}\right) \leq 1$. This implies that $C\left(v_{6}\right) \geq 3$. Clearly we are done if $C\left(v_{5}\right)=1$. Otherwise, we get $C\left(v_{6}\right) \geq 4$ and hence we can move two pebbles to $v_{3}$ from $v_{6}$ and $v_{4}$. Thus we can move one pebble to $v_{2}$.

Assume $C\left(v_{4}\right) \leq 1$. Similarly, we assume $C\left(v_{8}\right) \leq 1$. Assume $C\left(v_{9}\right) \geq 2$. Clearly, we are done if $C\left(v_{9}\right) \geq 4$ or $C\left(v_{3}\right)=1$ or $C\left(v_{1}\right)=1$. Assume $C\left(v_{3}\right)=0$ or $C\left(v_{1}\right)=0$. Let $C\left(v_{9}\right)=2$ or 3 . Since either $C\left(v_{6}\right)+C\left(v_{5}\right) \geq 4$ or $C\left(v_{6}\right)+C\left(v_{7}\right) \geq 4$, we can move one pebble to either $v_{3}$ or $v_{1}$. Since $C\left(v_{9}\right) \geq 2$, we can move another one pebble to $v_{1}$ or $v_{3}$ and hence we can move one pebble to $v_{2}$. So, we assume $C\left(v_{9}\right) \leq 1$. Also, we must have $C\left(v_{5}\right) \leq 1$ and $C\left(v_{7}\right) \leq 1$ (otherwise one pebble could be moved to $v_{2}$ ). Thus we have $C\left(v_{6}\right) \geq 4$. Clearly, we are done if $C\left(v_{1}\right)=1$ or $C\left(v_{3}\right)=1$. Assume $C\left(v_{1}\right)=0$ and $C\left(v_{3}\right)=0$. Thus we get $C\left(v_{6}\right) \geq 6$. Clearly, we are done if $C\left(v_{5}\right)=1$ or $C\left(v_{7}\right)=1$. Assume $C\left(v_{5}\right)=0$ and $C\left(v_{7}\right)=0$. Now, we have $C\left(v_{6}\right) \geq 8$ and hence we can move one pebble to $v_{2}$ easily.

So, the result is true for $t=1$. Assume the result is true for $t^{\prime} \geq 2$. Now consider the distribution of $8(t-1)+11$ pebbles on the vertices of $L_{4}$. Clearly, we can move one pebble to any target vertex $v_{i}$ at a cost of at most eight pebbles, since $C\left(L_{4}\right) \geq 19$ and $f\left(L_{4}\right)=11$. After moving one pebble to $v_{i}$, the remaining number of pebbles on the vertices of $L_{4}$ is at least $8(t-2)+11$. Hence we can move the additional $t-1$ pebbles to $v_{i}$, by induction. Thus $f_{t}\left(L_{4}\right) \leq 8(t-1)+11$.

Theorem 2.6. For $L_{5}$, the $t$-pebbling number is $f_{t}\left(L_{5}\right)=8(t-1)+13$.

Proof. Let $C\left(v_{8}\right)=8(t-1)+7, C\left(v_{4}\right)=C\left(v_{5}\right)=C\left(v_{6}\right)=C\left(v_{10}\right)=$ $C\left(v_{11}\right)=1$ and $C\left(v_{i}\right)=0$ for all $i \neq 4,5,6,8,10,11$. Then we cannot move $t$ pebbles to $v_{2}$. Thus $f_{t}\left(L_{5}\right) \geq 8(t-1)+13$.

We have three cases to prove $f\left(L_{5}\right) \leq 13$. Consider the distribution of 13 pebbles on the vertices of $L_{5}$.

Case 1: Let $v_{11}$ be the target vertex.

Clearly, $C\left(v_{11}\right)=0$ and $C\left(v_{i}\right) \leq 1$ for all $v_{i} \in S_{1}$ by Remark 1.9. Since, $C\left(S_{2}\right) \geq 8$, there exists a vertex, say $v_{2}$, such that $C\left(v_{2}\right) \geq 2$. Clearly, we are done if $C\left(v_{2}\right) \geq 4$. If $C\left(v_{1}\right)=1$ or $C\left(v_{3}\right)=1$ or $C\left(v_{4}\right) \geq 2$ or $C\left(v_{10}\right) \geq 2$ then we can move one pebble to $v_{11}$ easily. Assume $C\left(v_{1}\right)=0$, 
$C\left(v_{3}\right)=0, C\left(v_{4}\right) \leq 1$, and $C\left(v_{10}\right) \leq 1$. Now, we can move one pebble to $v_{11}$ easily, since $C\left(v_{6}\right) \geq 4$ or $C\left(v_{8}\right) \geq 4$.

Case 2: Let $v_{1}$ be the target vertex.

Clearly, $C\left(v_{1}\right)=0, C\left(v_{2}\right) \leq 1, C\left(v_{3}\right) \leq 1, C\left(v_{9}\right) \leq 1, C\left(v_{10}\right) \leq 1$ and $C\left(v_{11}\right) \leq 1$, by Remark 1.9. Let $C\left(v_{5}\right) \geq 2$. If $C\left(v_{5}\right) \geq 4$, then clearly we are done. Assume $C\left(v_{5}\right)=2$ or 3 . If $C\left(v_{3}\right)=1$ or $C\left(v_{11}\right)=1$ or $C\left(v_{4}\right) \geq 2$ or $C\left(v_{7}\right) \geq 2$ then we can move one pebble to $v_{1}$ easily. So, we assume $C\left(v_{3}\right)=0$ or $C\left(v_{11}\right)=0$ or $C\left(v_{4}\right) \geq 1$ or $C\left(v_{7}\right) \geq 1$. Then we have $C\left(v_{6}\right)+C\left(v_{8}\right) \geq 5$. Clearly we can move one pebble to $v_{11}$ using the pebbles at $v_{6}$ and $v_{8}$ and also we move one more pebble to $v_{11}$ from $v_{5}$. Thus we can move one pebble to $v_{1}$ through $v_{11}$. Assume $C\left(v_{5}\right) \leq 1$. In a similar way, we assume $C\left(v_{7}\right) \leq 1$. Now, we have $C\left(v_{4}\right)+C\left(v_{6}\right)+C\left(v_{8}\right) \geq 6$. Assume $C\left(v_{4}\right) \geq 2$. Clearly, we are done if $C\left(v_{4}\right) \geq 4$. Let $C\left(v_{4}\right)=2$ or 3 . Clearly, we are done if $C\left(v_{3}\right)=1$ or both $C\left(v_{5}\right)=C\left(v_{11}\right)=1$; if not then we have either $C\left(v_{6}\right) \geq 4$ or $C\left(v_{8}\right) \geq 4$. In either cases, we can move one pebble to $v_{1}$. So, we assume $C\left(v_{4}\right) \leq 1$. In a similar way, we assume that $C\left(v_{7}\right) \leq 1$. Thus we have $C\left(v_{6}\right) \geq 4$. Clearly, we are done if $C\left(v_{3}\right)=1$ or $C\left(v_{9}\right)=1$ or $C\left(v_{11}\right)=1$. Assume $C\left(v_{3}\right)=C\left(v_{9}\right)=C\left(v_{11}\right)=0$. Now we have $C\left(v_{6}\right) \geq 7$. We are done if $C\left(v_{5}\right)=1$ or $C\left(v_{7}\right)=1$. If not, then we get $C\left(v_{6}\right) \geq 8$ and hence we can move one pebble to $v_{1}$.

Case 3: Let $v_{2}$ be the target vertex.

Clearly, $C\left(v_{2}\right)=0, C\left(v_{1}\right) \leq 1$ and $C\left(v_{3}\right) \leq 1$, by Remark 1.9. Let $C\left(v_{4}\right) \geq$ 2. If $C\left(v_{4}\right) \geq 4$ then clearly we are done. Assume $C\left(v_{4}\right)=2$ or 3 then clearly $C\left(v_{3}\right)=0, C\left(v_{5}\right) \leq 1$ and $C\left(v_{11}\right) \leq 1$ (otherwise, we can move one pebble to $\left.v_{2}\right)$. If $C\left(v_{6}\right) \geq 4$ or $C\left(v_{7}\right) \geq 4$ or both $C\left(v_{6}\right) \geq 2$ and $C\left(v_{7}\right) \geq 2$ then we can move two pebbles to $v_{3}$ and then we move one pebble to $v_{2}$ from $v_{3}$. So, we assume $C\left(v_{6}\right)+C\left(v_{7}\right) \leq 4$ such that we cannot move one pebble to $v_{3}$. Let $C\left(v_{6}\right) \geq 2$. Clearly we are done if $C\left(v_{5}\right)=1$ or both $C\left(v_{7}\right)=1$ and $C\left(v_{11}\right)=1$. Otherwise, we get $C\left(v_{8}\right)+C\left(v_{9}\right)+C\left(v_{10}\right) \geq 5$. Clearly, we are done if $C\left(v_{9}\right) \geq 4$ or $C\left(v_{10}\right) \geq 4$ or both $C\left(v_{9}\right) \geq 2$ and $C\left(v_{10}\right) \geq 2$. Assume $C\left(v_{10}\right) \geq 2$. If $C\left(v_{1}\right)=1$ then we can move one pebble to $v_{2}$ easily. Let $C\left(v_{1}\right)=0$. Assume $C\left(v_{7}\right)=1$ and $C\left(v_{11}\right)=0$. We can move one pebble to $v_{9}$. Clearly we can move one pebble to $v_{1}$ through $v_{9}$, since either $C\left(v_{9}\right)=1$ or $C\left(v_{8}\right) \geq 2$ and hence one pebble could be moved to $v_{2}$. Now, assume $C\left(v_{7}\right)=0$ and $C\left(v_{11}\right)=1$. Clearly we can move one pebble to $v_{11}$ from the pebbles at the vertices $v_{6}$ and $v_{8}$ and hence one pebble could be moved to $v_{1}$. Then we can move one pebble to $v_{2}$ easily. 
Assume $C\left(v_{7}\right)=C\left(v_{11}\right)=0$. Now we have $C\left(v_{8}\right)+C\left(v_{9}\right) \geq 4$ and hence we can move one pebble to $v_{1}$. Thus we can move one pebble to $v_{2}$ easily. Assume $C\left(v_{10}\right) \leq 1$. In a similar way, we assume that $C\left(v_{9}\right) \leq 1$. Clearly, $C\left(v_{8}\right) \geq 3$. We can move one pebble to $v_{11}$ or $v_{1}$. Thus we are done if $C\left(v_{1}\right)=1$ or $C\left(v_{11}\right)=1$. Otherwise, we get $C\left(v_{8}\right) \geq 4$. Thus we move one pebble to $v_{5}$ from $v_{8}$ and hence we are done. Thus we can move one pebble to $v_{2}$ when $C\left(v_{6}\right) \geq 2$. So we assume that $C\left(v_{6}\right) \leq 1$. In a similar way, we could assume that $C\left(v_{7}\right) \leq 1$. Again we get $C\left(v_{8}\right)+C\left(v_{9}\right)+C\left(v_{10}\right) \geq 5$. By our previous discussions, we could send one pebble to $v_{1}$ or $v_{11}$ easily. Clearly, we are done if $C\left(v_{1}\right)=1$ or $C\left(v_{11}\right)=1$. Assume $C\left(v_{1}\right)=0$ and $C\left(v_{11}\right)=0$. Either we can move one pebble to $v_{3}$ or two pebbles to $v_{1}$ from the remaining distributions when $C\left(v_{4}\right)=2$ or 3 . So, we assume that $C\left(v_{4}\right) \leq 1$. In a similar way, we can assume that $C\left(v_{10}\right) \leq 1, C\left(v_{5}\right) \leq 1$ and $C\left(v_{9}\right) \leq 1$. Assume $C\left(v_{11}\right) \geq 2$. We can move one pebble to $v_{2}$ easily, if $C\left(v_{11}\right)=2$ or 3 or $C\left(v_{11}\right) \geq 4$. Assume $C\left(v_{11}\right) \leq 1$. Now, we have $C\left(v_{8}\right)+C\left(v_{9}\right) \geq 5$. Clearly, we can move one pebble to $v_{2}$ if $C\left(v_{1}\right)=1$ or both $C\left(v_{11}\right)=1$ and $C\left(v_{3}\right)=1$. If not, we have $C\left(v_{8}\right)+C\left(v_{9}\right) \geq 7$. We can move one pebble to $v_{2}$ easily except the distribution $C\left(v_{8}\right)=7$ and $C\left(v_{9}\right)=0$. We have either $C\left(v_{7}\right)=C\left(v_{11}\right)=1$ or $C\left(v_{7}\right)=C\left(v_{3}\right)=1$. Hence we can move one pebble to $v_{2}$ for this distribution too. Thus we can always move one pebble to $v_{2}$.

So, the result is true for $t=1$. Assume the result is true for $t^{\prime} \geq 2$. Now consider the distribution of $8(t-1)+13$ pebbles on the vertices of $L_{5}$. Clearly, we can move one pebble to any target vertex $v_{i}$ at a cost of at most eight pebbles, since $C\left(L_{5}\right) \geq 21$ and $f\left(L_{5}\right)=13$. After moving one pebble to $v_{i}$, the remaining number of pebbles on the vertices of $L_{5}$ is at least $8(t-2)+13$. Hence we can move the additional $t-1$ pebbles to $v_{i}$, by induction. Thus $f_{t}\left(L_{5}\right) \leq 8(t-1)+13$.

Theorem 2.7. For $L_{6}$, the $t$-pebbling number is $f_{t}\left(L_{6}\right)=16(t-1)+20$.

Proof. $\quad$ Let $C\left(v_{8}\right)=16(t-1)+15, C\left(v_{4}\right)=C\left(v_{6}\right)=C\left(v_{10}\right)=C\left(v_{12}\right)=1$ and $C\left(v_{i}\right)=0$ for all $i \neq 4,6,8,10,12$. Then we cannot move $t$ pebbles to $v_{2}$. Thus $f_{t}\left(L_{6}\right) \geq 16(t-1)+20$. We have three cases to prove $f\left(L_{6}\right) \leq 20$. Consider the distribution of 20 pebbles on the vertices of $L_{6}$.

Case 1: Let $v_{13}$ be the target vertex. 
Clearly, $C\left(v_{13}\right)=0$ and $C\left(v_{i}\right) \leq 1$ for all $v_{i} \in S_{1}$ by Remark 1.9. Since, $C\left(S_{2}\right) \geq 14$, there exists a vertex, say $v_{2}$, such that $C\left(v_{2}\right) \geq 3$. Clearly, we are done if $C\left(v_{2}\right) \geq 4$. If $C\left(v_{1}\right)=1$ or $C\left(v_{3}\right)=1$ or $C\left(v_{4}\right) \geq 2$ or $C\left(v_{12}\right) \geq 2$ then we can move one pebble to $v_{13}$ easily. Assume $C\left(v_{1}\right)=0$, $C\left(v_{3}\right)=0, C\left(v_{4}\right) \leq 1$, and $C\left(v_{12}\right) \leq 1$. Now, we can move one pebble to $v_{13}$ easily, since $C\left(v_{6}\right) \geq 4$ or $C\left(v_{8}\right) \geq 4$ or $C\left(v_{10}\right) \geq 4$.

Case 2: Let $v_{1}$ be the target vertex.

Clearly, $C\left(v_{1}\right)=0$ and $C\left(v_{i}\right) \leq 1$ for all $i \in\{2,3,11,12,13\}$ by Remark 1.9. Let $C\left(v_{5}\right) \geq 2$. If $C\left(v_{3}\right)=1$ or $C\left(v_{13}\right)=1$ or a vertex of $S_{1}-\left\{v_{1}, v_{3}, v_{11}\right\}$ has more than one pebble then we can move one pebble to $v_{1}$ easily. Otherwise, there exists a vertex, say $v_{6}$, of $S_{2}-\left\{v_{2}, v_{12}\right\}$, contains more than three pebbles and hence we are done. Assume $C\left(v_{i}\right) \leq 1$ for all $v_{i} \in S_{1}-\left\{v_{1}, v_{3}, v_{11}\right\}$. Clearly, $S_{2}-\left\{v_{2}, v_{12}\right\} \geq 13$, and so we can move two pebbles to $v_{13}$ and hence we are done.

Case 3: Let $v_{2}$ be the target vertex.

Clearly, $C\left(v_{2}\right)=0, C\left(v_{1}\right) \leq 1$ and $C\left(v_{3}\right) \leq 1$ by Remark 1.9. If $C\left(v_{5}\right) \geq 4$ then clearly we are done. So, we assume that $C\left(v_{5}\right) \leq 3$. Similarly, we assume that $C\left(v_{11}\right) \leq 3$. Let $C\left(v_{7}\right) \geq 4$. If $C\left(v_{1}\right)=1$ or $C\left(v_{3}\right)=1$ or $C\left(v_{4}\right) \geq 2$ or $C\left(v_{5}\right) \geq 2$ or $C\left(v_{11}\right) \geq 2$ or $C\left(v_{12}\right) \geq 2$ or $C\left(v_{13}\right) \geq 2$ then we can move one pebble to $v_{2}$ easily. Assume that $C\left(v_{1}\right)=0, C\left(v_{3}\right)=0$, $C\left(v_{4}\right) \leq 1, C\left(v_{5}\right) \leq 1, C\left(v_{11}\right) \leq 1, C\left(v_{12}\right) \leq 1$ and $C\left(v_{13}\right) \leq 1$. Also we can move one pebble to $v_{2}$ if $C\left(v_{9}\right) \geq 4$. So, we assume $C\left(v_{9}\right) \leq 3$. Let $C\left(v_{7}\right)=6$ or 7 . Clearly we are done if $C\left(v_{9}\right) \geq 2$ or $C\left(v_{6}\right) \geq 2$ or $C\left(v_{13}\right)=1$ or $C\left(v_{5}\right)=1$. So, we assume that $C\left(v_{9}\right) \leq 1, C\left(v_{6}\right) \leq 1, C\left(v_{13}\right)=0$ and $C\left(v_{5}\right)=0$. This implies that, we have both $C\left(v_{8}\right) \geq 2$ and $C\left(v_{10}\right) \geq 2$ and hence we can move one pebble to $v_{2}$ by moving four pebbles to $v_{13}$ using the pebbles at the vertices $v_{7}, v_{8}$ and $v_{10}$. Let $C\left(v_{7}\right)=4$ or 5 . Assume $C\left(v_{9}\right)=2$ or 3 . Clearly we are done if $C\left(v_{11}\right)=1$ or $p\left(v_{13}\right)=1$. Now, we have $C\left(v_{6}\right)+C\left(v_{8}\right)+C\left(v_{10}\right) \geq 10$. Clearly, we are done if $C\left(v_{6}\right) \geq 4$ or $C\left(v_{10}\right) \geq 4$. If not, then we have $C\left(v_{8}\right) \geq 4$. If $C\left(v_{6}\right) \geq 2$, then we move one pebble to $v_{5}$ and then we move three more pebbles to $v_{5}$ from the vertices $v_{7}$ and $v_{8}$ and hence we are done. So, we assume that $C\left(v_{6}\right) \leq 1$ and also $C\left(v_{10}\right) \leq 1$ in a similar way. Thus we have $C\left(v_{8}\right) \geq 8$ and hence we can one pebble to $v_{2}$ by moving four pebbles to $v_{13}$ using the pebbles at the vertices $v_{7}, v_{8}$. Now we assume that $C\left(v_{7}\right) \leq 3$. In a similar way, we assume that $C\left(v_{9}\right) \leq 3$. If four vertices of $S_{1}-\left\{v_{1}, v_{3}\right\}$ have two or more 
pebbles each then clearly we can move four pebbles to $v_{13}$ and hence one pebble can be moved to $v_{2}$ from $v_{13}$.

Subcase 3.1: Three vertices of $S_{1}-\left\{v_{1}, v_{3}\right\}$ have two or more pebbles each.

Clearly we are done if $C\left(v_{1}\right)=1$ or $C\left(v_{3}\right)=1$ or $C\left(v_{13}\right)=1$ or $C\left(v_{4}\right) \geq 2$ or $C\left(v_{5}\right) \geq 2$ or $C\left(v_{11}\right) \geq 2$ or $C\left(v_{12}\right) \geq 2$. Assume $C\left(v_{1}\right)=$ $C\left(v_{3}\right)=C\left(v_{13}\right)=0$ and $C\left(v_{4}\right) \leq 1, C\left(v_{5}\right) \geq 2, C\left(v_{11}\right) \geq 2$, and $C\left(v_{12}\right) \leq 1$. Clearly, $C\left(v_{6}\right)+C\left(v_{8}\right)+C\left(v_{10}\right) \geq 9$ and hence we can move one pebble to $v_{13}$ from the vertices $v_{6}, v_{8}$ and $v_{10}$. Thus we can move one pebble to $v_{2}$ using the pebbles at the three vertices of $S_{1}-\left\{v_{1}, v_{3}\right\}$.

Subcase 3.2: Two vertices of $S_{1}-\left\{v_{1}, v_{3}\right\}$ have two or more pebbles each. Clearly, we are done if $C\left(v_{1}\right)=1$ or $C\left(v_{3}\right)=1$ or $C\left(v_{4}\right) \geq 2$ or $C\left(v_{5}\right) \geq 2$ or $C\left(v_{11}\right) \geq 2$ or $C\left(v_{12}\right) \geq 2$ or $C\left(v_{13}\right) \geq 2$. Let $C\left(v_{13}\right)=1$ and so we can move three pebbles to $v_{13}$ from the two vertices of $S_{1}-\left\{v_{1}, v_{3}\right\}$ and $v_{6}, v_{8}$ and $v_{10}$. Assume $C\left(v_{13}\right)=0$ and so $C\left(v_{6}\right)+C\left(v_{8}\right)+C\left(v_{10}\right) \geq 11$. Thus we can move two pebbles to $v_{13}$ from the vertices $v_{6}, v_{8}$ and $v_{10}$ and then we move two more pebbles to $v_{13}$ from the two vertices of $S_{1}-\left\{v_{1}, v_{3}\right\}$ and hence we are done.

Subcase 3.3: One vertex of $S_{1}-\left\{v_{1}, v_{3}\right\}$ has two or more pebbles.

Clearly, we are done if $C\left(v_{1}\right)=1$ or $C\left(v_{3}\right)=1$ or $C\left(v_{4}\right) \geq 2$ or $C\left(v_{5}\right) \geq 2$ or $C\left(v_{11}\right) \geq 2$ or $C\left(v_{12}\right) \geq 2$ or $C\left(v_{13}\right) \geq 2$. Let $C\left(v_{13}\right)=1$ and so $C\left(v_{6}\right)+C\left(v_{8}\right)+C\left(v_{10}\right) \geq 12$. Thus we can move three pebbles to $v_{13}$ from the vertex of $S_{1}-\left\{v_{1}, v_{3}\right\}$ and the vertices $v_{6}, v_{8}$ and $v_{10}$. Assume $C\left(v_{13}\right)=0$ and let $v_{5}$ is the vertex of $S_{1}-\left\{v_{1}, v_{3}\right\}$ contains more than one pebble on it. So $C\left(v_{6}\right)+C\left(v_{8}\right)+C\left(v_{10}\right) \geq 13$. If $C\left(v_{7}\right)=1$ or $C\left(v_{9}\right)=1$ then we can move three pebbles to $v_{13}$ from $v_{6}, v_{8}$ and $v_{10}$ and hence we are done since $C\left(v_{5}\right) \geq 2$. Assume $C\left(v_{7}\right)=C\left(v_{9}\right)=0$ and so we can move three pebbles to $v_{13}$ from $v_{6}, v_{8}$ and $v_{10}$ and hence we are done. In a similar way, we can move one pebble to $v_{2}$ if $C\left(v_{11}\right) \geq 2, C\left(v_{7}\right) \geq 2$ and $C\left(v_{9}\right) \geq 2$.

Subcase 3.4: No vertex of $S_{1}-\left\{v_{1}, v_{3}\right\}$ has two or more pebbles. Clearly, we are done if $C\left(v_{1}\right)=1$ or $C\left(v_{3}\right)=1$ or $C\left(v_{4}\right) \geq 2$ or $C\left(v_{5}\right) \geq 2$ or $C\left(v_{11}\right) \geq 2$ or $C\left(v_{12}\right) \geq 2$ or $C\left(v_{13}\right) \geq 2$. Thus we have $C\left(v_{6}\right)+$ $C\left(v_{8}\right)+C\left(v_{10}\right) \geq 14$. Let $C\left(v_{13}\right)=1$. Clearly we can move three pebbles to $v_{13}$ if $C\left(v_{7}\right)=1$ or $C\left(v_{9}\right)=1$. Assume $C\left(v_{7}\right)=C\left(v_{9}\right)=0$ and so 
we can move three pebbles to $v_{13}$ since $C\left(v_{6}\right)+C\left(v_{8}\right)+C\left(v_{10}\right) \geq 15$ and hence we are done. Assume $C\left(v_{13}\right)=0$. Without loss of generality, we let $C\left(v_{6}\right) \geq 5$. If $C\left(v_{4}\right)=1$ or $C\left(v_{5}\right)=1$ or $C\left(v_{7}\right)=1$ then we can move two pebbles to $v_{3}$ and hence we are done. Assume $C\left(v_{4}\right)=C\left(v_{5}\right)=$ $C\left(v_{7}\right)=0$. Let $C\left(v_{8}\right) \geq 2$. If $C\left(v_{9}\right)=1$ then we move one pebble to $v_{13}$ and then we move another three pebbles to $v_{13}$ from $v_{6}, v_{8}$ and $v_{10}$ since $C\left(v_{6}\right)+C\left(v_{8}\right)+C\left(v_{10}\right)-2 \geq 16$ and hence we are done. Assume $C\left(v_{9}\right)=0$ and so $C\left(v_{6}\right)+C\left(v_{8}\right)+C\left(v_{10}\right) \geq 20$. Clearly we can move one pebble to $v_{2}$ from $v_{6}, v_{8}$ and $v_{10}$. So, the result is true for $t=1$. Assume the result is true for $t^{\prime} \geq 2$. Now consider the distribution of $16(t-1)+20$ pebbles on the vertices of $L_{6}$. Clearly, we can move one pebble to any target vertex $v_{i}$ at a cost of at most sixteen pebbles, since $C\left(L_{6}\right) \geq 36$ and $f\left(L_{6}\right)=20$. After moving one pebble to $v_{i}$, the remaining number of pebbles on the vertices of $L_{6}$ is at least $16(t-2)+20$. Hence we can move the additional $t-1$ pebbles to $v_{i}$, by induction. Thus $f_{t}\left(L_{6}\right) \leq 16(t-1)+20$.

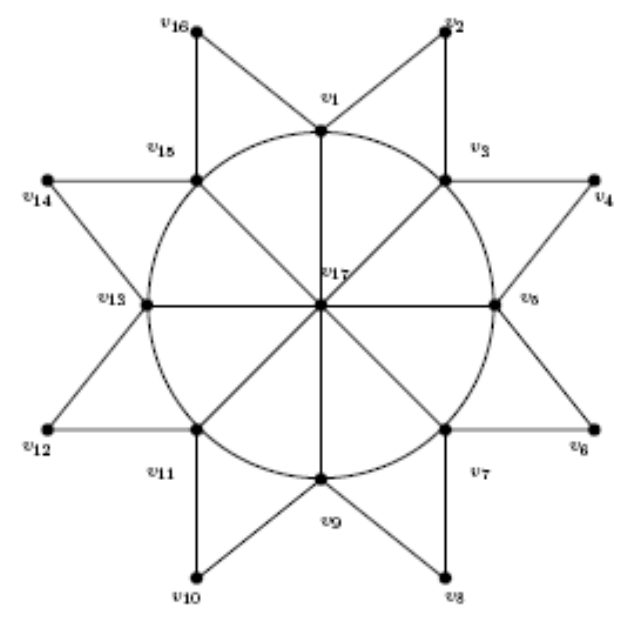

Figure 2. The Lamp graph $L_{8}$.

Theorem 2.8. For $L_{7}$, the t-pebbling number is $f_{t}\left(L_{7}\right)=16(t-1)+23$.

Proof. Let $C\left(v_{8}\right)=16(t-1)+15, C\left(v_{4}\right)=C\left(v_{6}\right)=C\left(v_{10}\right)=C\left(v_{14}\right)=$ $1, C\left(v_{12}\right)=3$ and $C\left(v_{i}\right)=0$ for all $i \neq 4,6,8,10,12,14$. Then we cannot move $t$ pebbles to $v_{2}$. Thus $f_{t}\left(L_{7}\right) \geq 16(t-1)+23$. Since $J_{2,7}$ is a spanning subgraph of $L_{7}$ and by Lemma 1 , we have $f\left(L_{7}\right) \leq f\left(J_{2,7}\right)=23$ (Theorem 
1.7). So the result is true for $t=1$. Assume the result is true for $t^{\prime} \geq 2$. Now consider the distribution of $16(t-1)+23$ pebbles on the vertices of $L_{7}$. Clearly, we can move one pebble to any target vertex $v_{i}$ at a cost of at most sixteen pebbles, since $C\left(L_{7}\right) \geq 39$ and $f\left(L_{7}\right)=23$. After moving one pebble to $v_{i}$, the remaining number of pebbles on the vertices of $L_{7}$ is at least $16(t-2)+23$. Hence we can move the additional $t-1$ pebbles to $v_{i}$, by induction. Thus $f_{t}\left(L_{7}\right) \leq 16(t-1)+23$.

Theorem 2.9. For $L_{n}(n \geq 8)$, the t-pebbling number is $f_{t}\left(L_{n}\right)=16(t-1)+2 n+10$.

Proof. Consider the following distribution for $L_{n}(n \geq 8)$ :

If $n$ is odd, let $C\left(v_{n+1}\right)=16(t-1)+15, C\left(v_{n-3}\right)=3, C\left(v_{n+5}\right)=3$, $C(x)=1$ where $x \notin N\left[v_{2}\right], x \notin N\left[v_{n+1}\right], x \notin N\left[v_{n-3}\right]$, and $x \notin N\left[v_{n+5}\right]$, and $C(y)=0$ where $y \in N\left[v_{2}\right], y \in N\left(v_{n+1}\right), y \in N\left(v_{n-3}\right)$, and $y \in N\left(v_{n+5}\right)$.

If $n$ is even, let $C\left(v_{n+2}\right)=16(t-1)+15, C\left(v_{n-2}\right)=3, C\left(v_{n+6}\right)=3$, $C(x)=1$ where $x \notin N\left[v_{2}\right], x \notin N\left[v_{n+2}\right], x \notin N\left[v_{n-2}\right]$, and $x \notin N\left[v_{n+6}\right]$, and $C(y)=0$ where $y \in N\left[v_{2}\right], y \in N\left(v_{n+2}\right), y \in N\left(v_{n-2}\right)$, and $y \in N\left(v_{n+6}\right)$.

Then, we cannot move a pebble to $v_{2}$. The total number of pebbles placed in both configurations are $16(t-1)+15+2(3)+(n-4)(1)+(n-8)(1)=$ $16(t-1)+2 n+9$. Therefore, $f_{t}\left(L_{n}\right) \geq 16(t-1)+2 n+10$.

Since $J_{2, n}$ is a spanning subgraph of $L_{n}(n \geq 8)$ and by Lemma 1 , we have $f\left(L_{n}\right) \leq f\left(J_{2, n}\right)=2 n+10$ (Theorem ??). So the result is true for $t=1$. Assume the result is true for $t^{\prime} \geq 2$. Now consider the distribution of $16(t-1)+2 n+10$ pebbles on the vertices of $L_{n}$. Clearly, we can move one pebble to any target vertex $v_{i}$ at a cost of at most sixteen pebbles, since $C\left(L_{n}\right) \geq 2 n+26$ and $f\left(L_{n}\right)=2 n+10$. After moving one pebble to $v_{i}$, the remaining number of pebbles on the vertices of $L_{n}$ is at least $16(t-2)+2 n+10$. Hence we can move the additional $t-1$ pebbles to $v_{i}$, by induction. Thus $f_{t}\left(L_{n}\right) \leq 16(t-1)+2 n+10$. 


\section{References}

[1] F. R. K. Chung, Pebbling in hypercubes, SIAM J. Disc. Math., 2 (4), pp. 467-472, (1989).

[2] P. Erdős, On pseudoprimes and Carmichael numbers, Publ. Math. Debrecen, 4, pp. 201-206, (1956).

[3] P. Erdős, A. Ginzburg and A. Ziv, A theorem in additive number theory, Bull. Res. Council Israel, 10F, pp. 41-43, (1961).

[4] D. Kleitman and P. Lemke, An addition theorem on the integers modulon, J. Number Theory, 31, pp. 335-345, (1989).

[5] A. Lourdusamy and T. Mathivanan, The t-pebbling number of Jahangir graph $J_{3, m}$, Proyecciones Journal of Mathematics, 34 (2), pp. 161-174, (2015).

[6] A. Lourdusamy and T. Mathivanan, The $t$-pebbling number of squares of cycles, Journal of Prime Research in Mathematics, 11, pp. 61-76, (2015).

[7] A. Lourdusamy, C. Muthulakshmi @ Sasikala and T. Mathivanan, The pebbling number of the square of an odd cycle, Sciencia Acta Xaveriana, 3 (2), pp. 21-38, (2012).

[8] A. Lourdusamy, F. Patrick and T. Mathivanan, The $t$-pebbling number of some wheel related graphs, Journal of Prime Research in Mathematics, 12, pp. 35-44, (2016).

[9] A. Lourdusamy, S. Samuel Jayaseelan and T. Mathivanan, On pebbling Jahangir graph, General Mathematics Notes, 5 (2), pp. 42-49, (2011).

[10] A. Lourdusamy, S. Samuel Jayaseelan and T. Mathivanan, Pebbling number for Jahangir graph $J_{2, m}(3 \leq m \leq 7)$, Sciencia Acta Xaveriana, 3 (1), pp. 87-106, (2012).

[11] A. Lourdusamy, S. Samuel Jayaseelan and T. Mathivanan, The tpebbling number of Jahangir graph, International Journal of Mathematical Combinatorics, 1, pp. 92-95, (2012). 
[12] L. Pachter, H.S. Snevily and B. Voxman, On pebbling graphs, Congressus Numerantium, 107, pp. 65-80, (1995).

\author{
A. Lourdusamy \\ Department of Mathematics, \\ St. Xavier's College (Autonomous) \\ Palayamkottai 627 002, \\ India \\ e-mail : lourdusamy15@gmail.com

\section{F. Patrick} \\ Department of Mathematics, \\ St. Xavier's College (Autonomous) \\ Palayamkottai 627 002, \\ India \\ e-mail : patrick881990@gmail.com \\ and

\section{T. Mathivanan} \\ Department of Mathematics, \\ St. Xavier's College (Autonomous) \\ Palayamkottai 627 002, \\ India \\ e-mail : tahit_van_man@yahoo.com
}

